\title{
Hydatid Disease of the Cervical Spine Mimicking Traumatic Burst Fracture
}

\author{
Babak Alijani ${ }^{{ }^{*}}$, Mohammadreza Emamhadi ${ }^{2}$, Shahrokh Yousefzadeh-Chabok ${ }^{3}$, Hamid Behzadnia ${ }^{1}$, \\ Siavash Dehghani 4
}

\author{
${ }^{1}$ MD, Assistant Professor of Neurosurgery, Poursina Hospital, Guilan University of Medical Sciences, Rasht, Iran \\ ${ }^{2}$ MD, Associate Professor of Neurosurgery, Poursina Hospital, Guilan University of Medical Sciences, Rasht, Iran \\ ${ }^{3}$ MD, Professor of Neurosurgery, Poursina Hospital, Guilan University of Medical Sciences, Rasht, Iran \\ ${ }^{4}$ MD, Senior Resident of Neurosurgery, Poursina Hospital, Guilan University of Medical Sciences, Rasht, Iran \\ * Corresponding Author Address: Poursina Hospital, Guilan University of Medical Sciences, Rasht, Guilan, Iran. Tel: +981333368773, \\ Fax: +981333339842, E-mail: babakalij@gmail.com
}

Article Type: Case Report

Received: November 28, 2015, Last revised: December 17, 2015, Accepted: February 3, 2016

\section{Abstract}

Background \& Importance: Hydatid disease of the spine is rare but constitutes approximately $50 \%$ of bone involvement cases in human hydatidosis. It is a severe condition associated with a high rate of morbidity, disability and mortality.

Case Presentation: In the present paper, we report an intriguing case of cervical spinal hydatid disease mimicking traumatic burst fracture.

Conclusion: A high degree of suspicion combined with good-quality neuroimaging is important for early and correct diagnosis. Because of the location and the potential for progression, spinal hydatid disease surgical treatment is the mainstay of treatment.

Keywords: Spine; Hydatid Disease; Echinococcosis

Please cite this paper as: Yousefzadeh-Chabok Sh, Alijani B, Emamhadi M, Behzadnia H, Dehghani S, Razzaghi A, Golmohammadi Sh. Hydatid Disease of the Cervical Spine Mimicking Traumatic Burst Fracture. Iran. J. Neurosurg. 2015;1(4):20-24.

\section{Background and Importance}

Hydatid disease or cystic echinococcosis, caused by the larval stage of the cestode Echinococcus granulosus, is a worldwide parasitic infection occurring on all continents except Antarctica (1). It is the most frequent in countries of the temperate zones, including the Mediterranean regions, Middle East, Indian subcontinent, southern and central parts of Russia, central Asia, China, Australia, New Zealand, South America, Baltic areas, and north and east Africa (2,3). Life cycle of Taenia echinococcus involves two mammalian hosts. Carnivores such as dogs, wolves and foxes are the definitive hosts, where the adult parasite lives. Intermediate hosts are herbivores like sheep, cattle and horses. They get infected when grazing on contaminated ground. Humans are occasionally accidentally infected by oral ingestion of tapeworm eggs existing in contaminated food or water or direct contact with host. After ingestion of the egg and digestion of the outer capsule, the larva penetrates the intestinal mucosa, enters into the host's circulatory system via venous and lymphatic pathways, and develops into the characteristic hydatid cyst (4-6). Hydatid cysts develops mainly in the liver $(65 \%)$ and the lungs $(25 \%)$, but any part of the body may be affected, including the muscles, spleen, bones, kidneys, brain, eyes, heart, and pancreas. Bone involvement is seen in $1 \%$ of all cases which almost half of them occur in spine. The central nervous system and the vertebral column are particularly vulnerable to the sequelae that result from their involvement. Involvement of the spinal cord, the spine, or both structures is associated with a high degree of morbidity, disability, and mortality (6-10). The prognosis is poor and this condition has often been compared to that of malignancies (white cancer) (11).

\section{Case Presentation}

A 48-year-old man visited the emergency room after experiencing a traffic car accident. On clinical evaluation, the patient had mild neck pain and tenderness, with no motor deficits or sensory deficits. Reflexes were normal and no signs of myelopathy or radiculopathy were detected. Lateral radiograph of the cervical spine showed C3 vertebral body destruction (Figure 1) and CT scan revealed irregular erosions of the cancellous bone devoid of any subperiosteal reaction (Figure 2). MRI demonstrated a detructive mass lesion with extension to spinal canal and cord compression (Figures $3 \& 4$ ).

The patient underwent a $\mathrm{C} 3$ corpectomy with total removal of the lesion and complete decompression of cervical canal. To reconstruct the vertebral body, a titanium mesh cage and an anterior cervical plate were used (Figure 5). The diagnosis of hydatid disease was confirmed histopathologically (Figure 6). After a consult with an infectious disease specialist, we discharged the patient with albendazole and advised him to continue treatment under observation of both services. Monthly visits were set. After 18 months of follow-up, he is doing great and no clinical symptoms or imaging signs of recurrence occurred yet. 


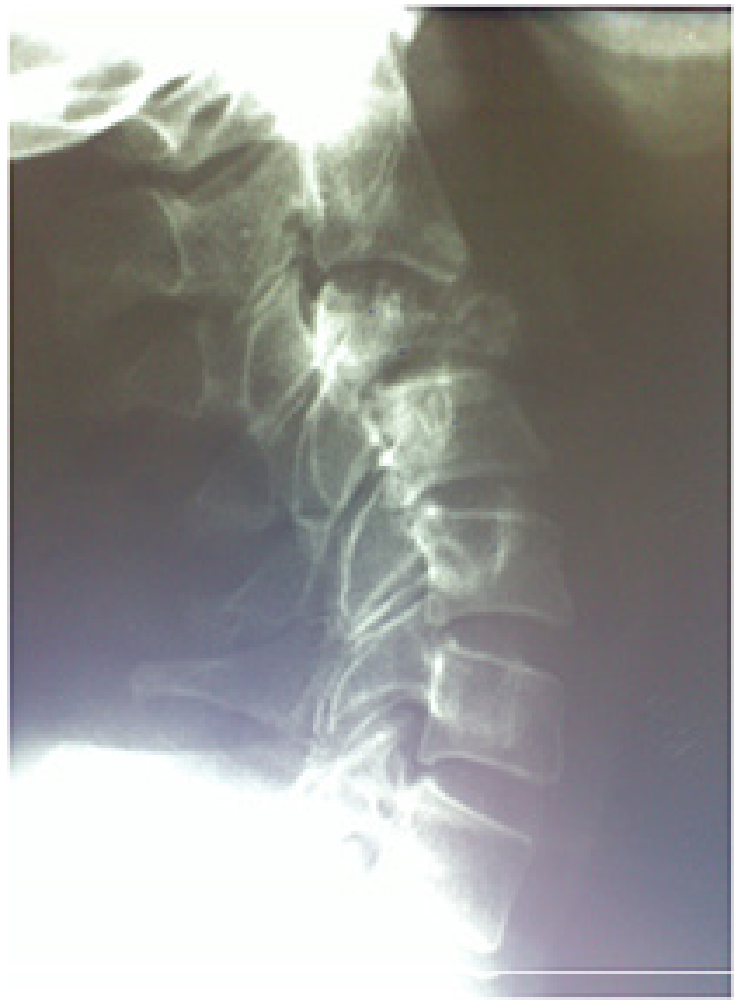

Figure 1. Lateral Radiograph of the Cervcal Spine Showing C3 Vertebral Body Destruction

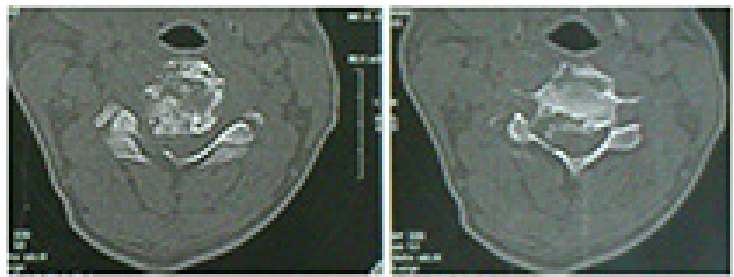

Figure 2. CT Scan Images Revealing Irregular Erosions of the Cancellous Bone Devoid of any Subperiosteal Reaction

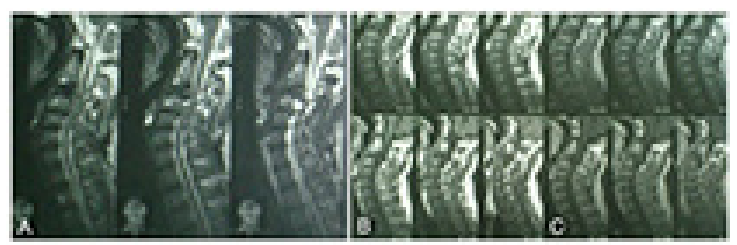

Figure 3. T2-weighted (A), T1-weighted (B) and Contrast Enhanced T1-weighted (C) MR Images Demonstrate a Destructive Mass Lesion with Spinal Cord Compression

\section{Discussion}

Many case series studies have reported the involvement of the thoracic (45-50\%) and the lumbar spine (20-39\%) in spinal hydatid disease $(12,13)$. Despite the vascular route of infection in spinal hydatid disease remains questionable, the predilection for the spine, and especially the thoracolumbar region, has been attributed to the dense local vascularization and the rich blood supply of the vertebral cancellous bone $(12,13)$. Paradoxic emboli through the Batson venous plexus, in which the blood flows directly from the intestinal vascular bed to the spine without being filtered through the liver or lungs, may be the reason for the occurrence of spine hydatidosis. Direct extension of a hepatic or pulmonary source, may be the other way of spine involvement $(3,14)$. In vertebral hydatid disease due to the resistant nature of bone, growth of the parasite is generally slow and characterized by aggressive bone infiltration. Pericyst formation does not occur in osseous hydatid disease and the microvesicular polycystic infiltration of the bone follows the line of least resistance along the intratrabecular spaces of the vertebra $(8,15,16)$. Local ischemic necrosis contributes to bone destruction in additon to the direct local pressure erosion of bone. Destructive growth eventually leads to symptomatic disease when the cysts transgress the vertebral cortex and infiltrate adjacent structures (like the spinal channel) or spontaneous fracture of the vertebra occurs. Intervertebral disc involvement in spinal hydatid disease is generally rare and the disc involvement is considered to be a late feature following extensive vertebral destruction in prolonged disease $(15,17)$. Intramural-extramedullary cysts usually grow eccentrically and the line of the least resistance along the dural sack is followed. Intradural hydatid disease is more frequently limited to a single cyst and infection appears to present at a younger age, than extradural lesion (18). Intramedullar hydatid disease is very rare and only three published cases are presented in the literature (19, 20). The presenting symptoms are not specific and vary with the location of the cyst. Spinal hydatid disease may manifest with any symptom due to vertebral bone destruction or spinal cord compression. A long history of back pain and/or subacute symptoms related to spinal cord or spinal nerve compression (radicular pain, peripheral sensitivity loss, sphincter disturbance, bladder dysfunction, paraparesis, paraplegia) are usually at the forefront of the clinical picture. The course of symptoms might range from acute onset to prolonged clinical courses, and the diagnosis is often only made many years or even decades after the first appearance of symptoms $(18,21)$. Correct diagnosis of hydatid disease is of significant importance, because suitable surgical planning and special intraoperative measures to prevent spillage, rely on preoperative suspicion $(1,22)$. The differential diagnosis consists of tuberculosis, pyogenic infections, mycoses, abscesses, fibrous dysplasia, enchondroma, metastatic malignancy, multiple myeloma, giant cell tumor, arachnoid cyst, and other benign or malignant neoplasms and osteolytic lesions which cause pathologic fractures $(1,23)$. Serodiagnostic tests such as detection of antibody directed to echinococcal polypeptide antigens, ELISA, indirect hemagglutination and complement fixation tests are reported to be $80-100 \%$ sensitive and $88-96 \%$ specific in abdominal disease. However, the sensitivity decreases abruptly to $25 \%$ to $56 \%$ in extrahepatic disease which limits their use in the diagnosis or follow up for primary bone disease and a negative serology should not exclude the diagnosis of hydatid disease in the presence of suggestive imaging results (3). The radiological approach of the spinal 


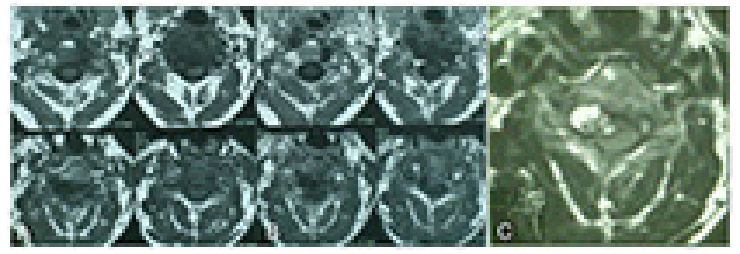

Figure 4. T1-weighted (A), Contrast Enhanced T1-weighted (B) and T2-weighted (C) MR Images Showing a Multi-loculated Lesion with Extension to the Cervical Canal

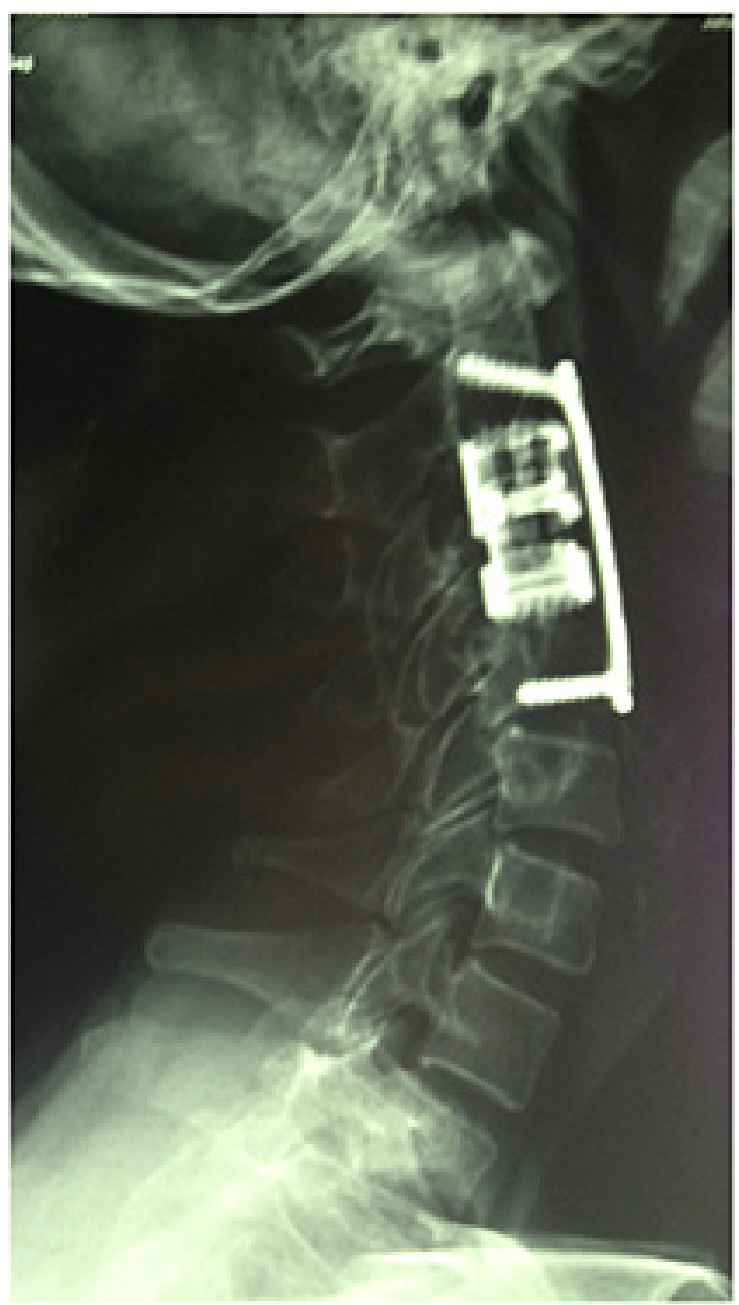

Figure 5. Post-operative Lateral Radiograph

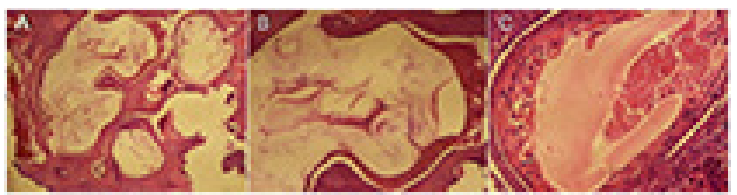

Figure 6. Hydatid Cyst Wall Infiltrating through Bony Trabeculae (A \& B) and in Some Areas is Surrounded by Inflammatory Cells (C) hydatid disease includes plain radiographs, CT scan, and MRI. On plain radiographs, multiple moth eaten lesions with surrounding sclerosis and calcifications in multiple contiguous vertebral bodies and appendages are seen, usually without vertebral collapse and with sparing of intervertebral disks. The bone destruction, is seen in the advanced stage. Involvement of contiguous ribs and paravertebral masses are suggestive of hydatid disease but the radiological features are not pathognomonic $(16,17,22)$. CT scan provides a precise assessment of the osseous part of the lesion and usually shows irregular erosions of the cancellous bone devoid of any subperiosteal reaction, enlargement of the vertebra, the extension into the soft tissues, and the calcifications of the peripheral rim of the cyst, if they exist. After administration of contrast agent, no enhancement is seen on CT scan imaging. Postmyelogram CT scan is useful when MRI is not available $(1,15,16,22)$. MRI technique is the method of choice and the most helpful tool in diagnosing spinal hydatid disease, determination of the extent of the disease, planning surgical approach, and early diagnosis of postoperative recurrence. MRI is more reliable than CT scan because it shows the typical signs of a pericystic wall and its multilocular character and has a special value in showing the relationship of the lesion to the surrounding organs. On MRI, the cysts have inhomogenous low signal intensity on T1-weighted images and very high intensity on T2-weighted images, and the cysts wall demonstrate slight contrast enhancement $(17,22)$. Spinal hydatidosis can be differentiated from abscesses or infected cystic lesions by diffusion-weighted imaging. The infected cystic lesions or abscesses are shown by presence of restricted diffusion.lesion (24). Since spinal hydatidosis is invasive by nature, successful treatment can be a challenge (22). Surgery remains the mainstay of treatment (1) and the two primary tasks in surgery of spinal hydatid disease are decompression of a compromised spinal cord and stabilization of a compromised spinal column (25). Preoperative medical treatment of the lesions that may be amenable to radical surgery is controversial. Medical treatment is the best option for benign slow-growing asymptomatic lesions, without risking an inadvertent surgical rupture, unless these lesions are in close proximity to the spinal cord. In recurrent infiltrative lesions, it is probably wise to combine protracted chemotherapy with punctuated palliative surgeries aimed to improve the quality of life of the patient (18). Cysts confined to the intradural space (Type 1\&2 according to the Dew/ Braithwaite \& Lees classification (16)) typically cause acute to subacute compression of the spinal cord and require urgent decompression. Intradural cysts are approached posteriorly via laminectomy followed by durotomy (18). Extraduralextraosseous cysts (Type 3\&5) range from single epidural or paravertebral cysts to large dumbbell formations and cases with extensive soft tissue infiltration. Epidural cysts and the majority of dumbbell cysts present with spinal cord compression and decompression by laminectomy is almost always part of the surgical management. Anterior approach is usually indicated to reach paravertebral lesions $(12,13$, 18). The infiltrative nature of the microvesicular lesions in vertebral hydatid disease (Type 4) does not allow for unruptured resection of the cysts and therefore spillage of their contents is inevitable $(13,15,18)$. Complete resection of the affected bone may be precluded in the extensive disease and progressive vertebral destruction may compromise stability of the spinal column (26). Radical surgery is the 
most common initial treatment, with total removal of the cysts being the primary goal. Although it often does not prohibit disease progression, but it has been shown that it prolongs survival in selected cases (27). Surgical approach is determined by the location and the extent of the lesion, and after resection of affected vertebra, a large variety of techniques have been used to stabilize the vertebral column $(15,18)$. Rupture of a cyst with spillage of its content in addition to recurrence may provoke various hypersensitivity reactions such as pruritus, urticaria, edema, dyspnea, bronchospasm, asthma, vomiting, diarrhea, colicky abdominal pain and even fatal anaphylactic shock (1, 28). Some authors have recommended the use of corticosteroids to prevent anaphylactic shock during surgery (8). If cysts rupture during surgery, irrigation of the field with scolicidal solutions is advocated by most surgeons, however, this seems relatively ineffective $(3,13)$. Several agents such as hypertonic saline, ethanol, povidone iodine, ethacrine lactate (rivanol), hydrogen peroxide, silver nitrate, cetrimide, and formalin have been deployed to irrigate the operative field (13). Scolocidal agents are not active against intact microscopic daughter cysts (which demand chemotherapy). They are considered as a valuable adjunct to radical surgery and chemotherapy, because of their effectiveness against spilled protoscolices from ruptured cysts (8). Therapy with benzimidazoles (mebendazole \& albendazole) is used preoperatively (if diagnosed) and postoperatively for the local control of disease, prevention of systemic spread and avoidance of recurrence. $(3,29)$ Albendazole is preferred over mebendazole due to better pharmacokinetic properties and superior efficacy against helminthes, and most authors use the dosages recommended by the World Health Organization (WHO) for visceral hydatid disease (mebendazole 40-50 $\mathrm{mg} / \mathrm{kg} /$ day; albendazole $10-15 \mathrm{mg} / \mathrm{kg} / \mathrm{day}$ ) (30). To achieve scolicidal blood levels, treatment with albendazole should be initiated $\geq 4$ hours before surgery, with regard to high to inevitable risk of cyst rupture (31). There is some evidence to support a role for the use of praziquantel in combination with albendazole in pre- and post-intervention medical treatment for hydatid disease. The risk of disease recurrence and seeding of infection may be reduced by combined therapy (32). There is no agreement on the optimal duration of medical treatment for various forms of spinal hydatid disease but most authors prefer 6 months of treatment (18).

\section{Conclusion}

Due to the nature of disease, suspicion in endemic countries is of high importance and hydatid disease should be considered as a differential diagnosis especially in pathologic fractures. Appropriate physical exams and adequate imaging studies to identify the disease must be taken into account. Familiarity with different aspects of hydatid disease imaging in an unusual location may be valuable in making a correct diagnosis and treatment. Surgery is the treatment of choice which is individualized to each patient according to the location of the lesion. Decompression of a compromised spinal cord and stabilization of a compromised spinal column are essential goals of surgery.

\section{Acknowledgement}

The authors offer their special thanks to the Clinical Research Development Unit of Poursina Hospital, Guilan University of Medical Sciences, Rasht, Guilan, Iran.

\section{Funding}

None

\section{Conflict of interests}

The authors declare no conflict of interests.

\section{References}

1. Pamir MN, Ozduman K, Elmaci I. Spinal hydatid disease. Spinal Cord. 2002;40(4):153-60.

2. Grosso G, Gruttadauria S, Biondi A, Marventano S, Mistretta A. Worldwide epidemiology of liver hydatidosis including the Mediterranean area. World J Gastroenterol. 2012;18(13):1425-37.

3. Lotfinia I, Sayyahmelli S, Mahdkhah A, Shoja M. Intradural extramedullary primary hydatid cyst of the spine: a case report and review of literature. European Spine Journal. 2013;22(3):329-36.

4. Çelik C, Şaşmaz MF, Uçan H. Spinal hydatid cyst: review. Türkiye Klinikleri Tıp Bilimleri Dergisi. 2010;30(3):1073-7.

5. Sultana N, Hashim TK, Jan SY, Malik T, Shah W. Primary cervical hydatid cyst: a rare occurrence. Diagnostic pathology. 2012;7(1):157.

6. Celik A, Turanli M, Kutun S, Delibasi T, Mengi N, Comert E, et al. Unusual location of hydatid cyst: soft tissue mass in the neck. European Archives of Oto-Rhino-Laryngology and Head \& Neck. 2006;263(12):1147-50.

7. Dagtekin A, Koseoglu A, Kara E, Karabag H, Avci E, Torun F, et al. Unusual location of hydatid cysts in pediatric patients. Pediatric neurosurgery. 2009;45(5):379-83.

8. Song X, Ding L. H Wen H. Bone hydatid disease Postgrad Med J. 2007;83:536-42.

9. Abbassioun K, Amirjamshidi A. Diagnosis and management of hydatid cyst of the central nervous system: Part 1: General considerations and hydatid disease of the brain. Neurosurgery Quarterly. 2001;11(1):1-9.

10. Kammerer WS, editor Echinococcosis affecting the central nervous system. Seminars in neurology; 1993.

11. DÉVÉ F. Hydatid Disease of the Bones. L'echinococcose osseuse. 1948.

12. Altinörs N, Bavbek M, Caner HH, Erdogan B. Central nervous system hydatidosis in Turkey: a cooperative study and literature survey analysis of 458 cases. Journal of neurosurgery. 2000;93(1):1-8.

13. Turgut M. Hydatid disease of the spine: a survey study from Turkey. Infection. 1997;25(4):221-6.

14. Lath R, Ratnam BG, Ranjan A. Diagnosis and treatment of multiple hydatid cysts at the craniovertebral junction: Case report. Journal of Neurosurgery: Spine. 2007;6(2):174-7.

15. Baysefer A, Gönül E, Canakci Z, Erdoğan E, Aydoğan N, Kayali H. Hydatid disease of the spine. Spinal Cord. 1996;34(5):297-300.

16. Braithwaite PA, Lees RF. Vertebral hydatid disease: radiological assessment. Radiology. 1981;140(3):763-6.

17. Tsitouridis I, Dimitriadis A. CT and MRI in vertebral hydatid disease. European radiology. 1997;7(8):1207-10.

18. Neumayr A, Tamarozzi F, Goblirsch S, Blum J, Brunetti E. Spinal cystic echinococcosis-a systematic analysis and review of the literature: part 2 . Treatment, follow-up and outcome. PLoS Negl Trop Dis. 2013;7(9):e2458.

19. Ley Jr A, Marti A. Intramedullary hydatid cyst: Case report. Journal of neurosurgery. 1970;33(4):457-9.

20. Senol MG, Tekeli HK, Mustafa Tansel K, Serdar TV, Güner S, Saracoglu M. Intramedullary hydatid cyst of the cervical spine. Indian Journal of Medical Microbiology.30(4).

21. Limaiem F, Bellil S, Bellil K, Chelly I, Mekni A, Khaldi M, et al. Primary hydatidosis of the central nervous system: a retrospective study of 39 Tunisian cases. Clinical neurology and neurosurgery. 2010;112(1):23-8.

22. Prabhakar MM, Acharya AJ, Modi DR, Jadav B. Spinal hydatid disease: a case series. The journal of spinal cord medicine. 2005;28(5):426.

23. Kalkan E, Torun F, Erdi F, Baysefer A. Primary lumbar vertebral hydatid cyst. Journal of Clinical Neuroscience. 2008;15(4):472-3.

24. Doganay S, Kantarci M. Role of conventional and diffusion-weighted magnetic resonance imaging of spinal treatment protocol for hydatid disease. The journal of spinal cord medicine. 2009;32(5):574

25. Chaudhary K, Bapat M, Badve S. Paraplegia due to recurrent multiple hydatid cyst of the spine: a case report. Indian journal of orthopaedics. 2008;42(2):222

26. Özdemir HM, Ögün TC, Tasbas B. A lasting solution is hard to achieve in primary hydatid disease of the spine: long-term results and an overview. Spine. 2004;29(8):932-7.

27. Turtas S, Viale ES, Pau A. Long-term results of surgery for hydatid disease of the spine. Surgical neurology. 1980;13(6):468-70.

28. Neumayr A, Troia G, De Bernardis C, Tamarozzi F, Goblirsch S, Picoli L, et al. Justified concern or exaggerated fear: the risk of anaphylaxis in percutaneous treatment of cystic echinococcosis - a systematic literature review. PLoS Neg1 Trop Dis. 2011;5(6):e1154.

29. Kotil K, Tari R, Savas Y. Medical treatment of primary extradural solitary lumbar hydatid disease. Journal of Clinical Neuroscience. 2010;17(6):793-5.

30. WHO Guidelines for treatment of cystic and alveolar 
echinococcosis in humans. WHO Informal Working Group on Echinococcosis. Bull World Health Organ. 1996;74(3):231-42.

31. Brunetti E, Kern P, Vuitton DA. Expert consensus for the diagnosis and treatment of cystic and alveolar echinococcosis in humans. Acta tropica. 2010;114(1):1-16.

32. Bygott J, Chiodini P. Praziquantel: neglected drug? Ineffective treatment? Or therapeutic choice in cystic hydatid disease? Acta tropica. 2009;111(2):95101. 